\title{
Medical Image Segmentation Based on Fractional-Order Derivative
}

\author{
Dan Tian \\ School of Information Engineering \\ Shenyang University \\ Shenyang, China \\ www.sltd2008@163.com
}

Dapeng Li

School of Information Engineering

Shenyang University

Shenyang, China

295677734@qq.com

\author{
Yingxin Zhang \\ School of Information Engineering \\ Shenyang University \\ Shenyang, China \\ 735497145@qq.com
}

\begin{abstract}
Objective: In the traditional edge detection differential operators, the first-order derivative masks are easy to loss image details information, and the second-order derivative masks are more sensitive to noise. As for these problems, this paper proposes a fractional-order mask for medical image segmentation. Methods: Combining the frequency characteristic and the memorability of fractional differential, the classical first-order Sobel operator is generalized to fractional-order mode; A fractional-order differential mask is constructed for extracting the edge feature of medical images. Results: The experiment results show that compared with the integer order differential mask, the fractional-order differential mask can detect more edge details feature of the medical images, and is more robust to noise. Conclusion: Based on the global characteristic of the fractional differential, the proposed fractional-order Sobel mask can extract more image edge feature details. Experiment results show that the proposed fractional-order mask yields good visual effects for brain MRI image segmentation.
\end{abstract}

Keywords- medical image; image segmentation; fractional-order; frequency characteristic; Sobel operator

\section{INTRODUCTION}

Medical image segmentation has important applications in medical image visualization, localization of pathology, and computer-aided diagnosis. Edge detection[1-3] is one of the most frequently used techniques in image segmentation. It primarily can be classified into two categories: first-order derivative and second-order derivative methods. However, the first-order derivative methods generally produce thicker edges, which results in the loss of image details. The second-order derivative methods have a stronger response to fine detail, but they are more sensitive to noise. To solve these problems, the fractional-order derivative has been introduced to the edge detection methods. Since the frequency characteristic determines it can preserve more low-frequency contour feature, keep high-frequency marginal feature and also enhance medium-frequency texture details. Considering this superiority, $\mathrm{Pu}$ et al. [4] proposed a class of fractional differential masks for image texture enhancement. Bai and Feng [5] proposed a class of fractional-order anisotropic diffusion models for noise removal. In this paper, we generalize the classical Sobel operator[6] to constitute a fractional-order Sobel operator for edge detection. The proposed operator is tested in brain MRI image segmentation. The results are promising compared to those of the first-order Sobel operator.

\section{DIFFERENTIAL MASK}

\section{A. Sobel mask}

Edges occur where there is a discontinuity in the intensity function or a very steep intensity gradient in the image. The gradient of an image $f(x, y)$ at $(x, y)$ can be defined as a vector

$$
\nabla f(x, y)=\left[G_{x}, G_{y}\right]^{T}
$$

where

$$
\left\{\begin{array}{l}
G_{x}=f(x+1, y)-f(x, y) \\
G_{y}=f(x, y+1)-f(x, y)
\end{array}\right.
$$

Sobel detection is a classical first-order derivative detection method, which brings out the horizontal and vertical edges individually based on a pair of $3 \times 3$ convolution operator shown in Fig.1.

\begin{tabular}{|c|c|c|}
\hline-1 & -2 & -1 \\
\hline 0 & 0 & 0 \\
\hline 1 & 2 & 1 \\
\hline
\end{tabular}

\begin{tabular}{|c|c|c|}
\hline-1 & 0 & 1 \\
\hline-2 & 0 & 2 \\
\hline-1 & 0 & 1 \\
\hline
\end{tabular}

Figure 1. Sobel mask

The operator can be applied separately to a $3 \times 3$ neighbourhood of the center point pixel. The gradient components along the $\mathrm{x}$ - and $\mathrm{y}$ - directions can be found using the following approximation 


$$
\begin{aligned}
G_{x}= & -f(x-1, y-1)+f(x+1, y-1)-2 f(x-1, y)+ \\
& 2 f(x+1, y)-f(x-1, y+1)+f(x+1, y+1) \\
G_{y}= & -f(x-1, y-1)+f(x-1, y+1)-2 f(x, y-1)+ \\
& 2 f(x, y+1)-f(x+1, y-1)+f(x+1, y+1)
\end{aligned}
$$

In discrete images, we can consider $\Delta x$ and $\Delta y$ in terms of numbers of pixel between two pixel points. Let $\Delta x=\Delta y=2$, then the differential form of the gradient components can be given by

$$
\begin{aligned}
& G_{x}=\frac{1}{2}\left(\frac{\partial f(x+1, y-1)}{\partial x}+2 \frac{\partial f(x+1, y)}{\partial x}+\frac{\partial f(x+1, y+1)}{\partial x}\right) \\
& G_{y}=\frac{1}{2}\left(\frac{\partial f(x-1, y+1)}{\partial y}+2 \frac{\partial f(x, y+1)}{\partial y}+\frac{\partial f(x+1, y+1)}{\partial y}\right)
\end{aligned}
$$

\section{B. Fractional-order Sobel mask}

Fractional-order derivative has been applied in various scientific fields including image processing [7-9].But the definition of the fractional-order derivative is not unified until now. In this paper, the Grünwald-Letnikov definition[10] is used. Assume the size of image $f$ is $M \times N$, then the discrete form of $\nabla^{\alpha} f$ can be represented as

$$
\left(\nabla^{\alpha} f\right)_{i, j}=\left(\left(\Delta_{1}^{\alpha} f\right)_{i, j},\left(\Delta_{2}^{\alpha} f\right)_{i, j}\right) \quad 1 \leq i \leq M, 1 \leq j \leq N
$$

with

$$
\left\{\begin{array}{l}
\left(\Delta_{1}^{\alpha} f\right)_{i, j}=\sum_{k=0}^{K-1}(-1)^{k} C_{k}^{\alpha} f_{i-k, j} \\
\left(\Delta_{2}^{\alpha} f\right)_{i, j}=\sum_{k=0}^{K-1}(-1)^{k} C_{k}^{\alpha} f_{i, j-k}
\end{array}\right.
$$

where $K \geq 3$ is an integer constant , $C_{k}^{\alpha}=\frac{\Gamma(\alpha+1)}{\Gamma(k+1) \Gamma(\alpha-k+1)}, \Gamma$ is the gamma function.

By generalizing the order from integer to fraction, a fractional-order differential form of the gradient components transformed from (5) and (6) can be obtained as

$$
\begin{aligned}
& G_{x}^{\alpha}=\frac{1}{2}\left(\frac{\partial^{\alpha} f(x+1, y-1)}{\partial x^{\alpha}}+2 \frac{\partial^{\alpha} f(x+1, y)}{\partial x^{\alpha}}+\frac{\partial^{\alpha} f(x+1, y+1)}{\partial x^{\alpha}}\right) \\
& G_{y}^{\alpha}=\frac{1}{2}\left(\frac{\partial^{\alpha} f(x-1, y+1)}{\partial y^{\alpha}}+2 \frac{\partial^{\alpha} f(x, y+1)}{\partial y^{\alpha}}+\frac{\partial^{\alpha} f(x+1, y+1)}{\partial y^{\alpha}}\right)
\end{aligned}
$$

\begin{tabular}{|c|c|c|c|c|}
\hline$(-1)^{k} C_{k}^{\alpha} / 2$ & \multicolumn{2}{|c|}{$(-1)^{k} C_{k}^{\alpha}$} & \multicolumn{2}{|c|}{$(-1)^{k} C_{k}^{\alpha} / 2$} \\
\hline$\vdots$ & \multicolumn{2}{|c|}{ : } & \multicolumn{2}{|l|}{$\vdots$} \\
\hline$\left(\alpha^{2}-\alpha\right) / 4$ & \multicolumn{2}{|c|}{$\left(\alpha^{2}-\alpha\right) / 2$} & \multicolumn{2}{|c|}{$\left(\alpha^{2}-\alpha\right) / 4$} \\
\hline$-\alpha / 2$ & \multicolumn{2}{|c|}{$-\alpha$} & \multicolumn{2}{|c|}{$-\alpha / 2$} \\
\hline $1 / 2$ & \multicolumn{2}{|c|}{1} & \multicolumn{2}{|l|}{$1 / 2$} \\
\hline$(-1)^{k} C_{k}^{\alpha}$ & $\ldots$. & \multirow{2}{*}{$\frac{\left(\alpha^{2}-\alpha\right)}{4}$} & $-\alpha$ & $\underline{1}$ \\
\hline 2 & & & 2 & 2 \\
\hline$(-1)^{k} C_{k}^{\alpha}$ & $\ldots$ & $\frac{\left(\alpha^{2}-\alpha\right)}{2}$ & $-\alpha$ & 1 \\
\hline$(-1)^{k} C_{k}^{\alpha}$ & $\ldots$ & $\left(\alpha^{2}-\alpha\right)$ & $-\alpha$ & 1 \\
\hline 2 & & 4 & 2 & 2 \\
\hline
\end{tabular}

Then the fractional-order gradient components along the $\mathrm{x}$ - and $\mathrm{y}$ - directions can be found using the following approximation

$$
\begin{aligned}
G_{x}^{\alpha}= & \frac{1}{2}\left[f(x+1, y-1)-\alpha f(x, y-1)+\frac{\alpha^{2}-\alpha}{2} f(x-1, y-1)\right. \\
& +\ldots+(-1)^{k} C_{k}^{\alpha} f(x+1-k, y-1)+2 f(x+1, y)-2 \alpha f(x, y)+ \\
& \left(\alpha^{2}-\alpha\right) f(x-1, y)+\ldots+2 *(-1)^{k} C_{k}^{\alpha} f(x+1-k, y)+ \\
& f(x+1, y+1)-\alpha f(x, y+1)+\frac{\alpha^{2}-\alpha}{2} f(x-1, y+1)+\ldots+ \\
& \left.(-1)^{k} C_{k}^{\alpha} f(x+1-k, y+1)\right]
\end{aligned}
$$

$$
\begin{aligned}
G_{y}^{\alpha}= & \frac{1}{2}\left[f(x-1, y+1)-\alpha f(x-1, y)+\frac{\alpha^{2}-\alpha}{2} f(x-1, y-1)\right. \\
& +\ldots+(-1)^{k} C_{k}^{\alpha} f(x-1, y+1-k)+2 f(x, y+1)- \\
& 2 \alpha f(x, y)+\left(\alpha^{2}-\alpha\right) f(x, y-1)+\ldots+ \\
& 2 *(-1)^{k} C_{k}^{\alpha} f(x, y+1-k)+f(x+1, y+1)- \\
& \alpha f(x+1, y)+\frac{\alpha^{2}-\alpha}{2} f(x+1, y-1)+\ldots+ \\
& \left.(-1)^{k} C_{k}^{\alpha} f(x+1, y+1-k)\right]
\end{aligned}
$$

According to the former approximation, we propose a fractional-order Sobel mask described as follows.

Figure 2. Fractional-order Sobel mask

From the definition, an important property can be observed. The integer derivative just involves finite terms; However, the fractional derivative involves infinite number of terms. Therefore, the integer derivative is a local operator while the fractional derivative is a global operator. The fractional mask can consider more neighboring pixels information, extracting more image details.

\section{SEGMENTATION THRESHOLDING}

An important quantity in edge detection is the gradient magnitude of the image, as for the fractional-order gradient, the magnitude measure can be denoted as

$$
\nabla^{\alpha} F=\operatorname{mag}\left(\nabla^{\alpha} f\right)=\left[\left(G_{x}^{\alpha}\right)^{2}+\left(G_{y}^{\alpha}\right)^{2}\right]^{1 / 2}
$$

This quantity gives the maximum rate of increase of $f(x, y)$ per unit distance in the direction of $\nabla^{\alpha} f$. In this paper, the thresholding is selected based on the average gradient,

$$
T=\tau \sum_{i=1}^{M} \sum_{j=1}^{N} \nabla^{\alpha} F /(M N)
$$

where $T$ denotes the thresholding, $\tau \geq 1$ is a predetermined parameter. Then the pixel can be marked as edge points if $\nabla^{\alpha} F>T$.

\section{NUMERICAL RESULTS}

In this section, we provide numerical results to show the behavior of the proposed fractional-order mask with respect to its edge extraction capability. 


\section{A. The selection of fractional order}

In the following, we will analysis the probelm of order selection in the fractional edge detection operator based on its frequency characteristic. Fig. 3 shows the amplitudefrequency response of differential, when $\alpha \in(0,2.0]$.

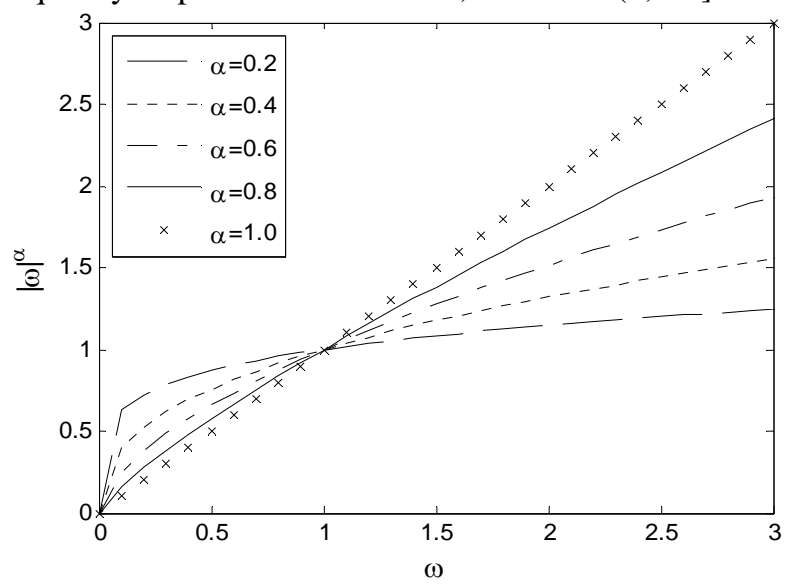

(a) $\alpha \in(0,1.0]$

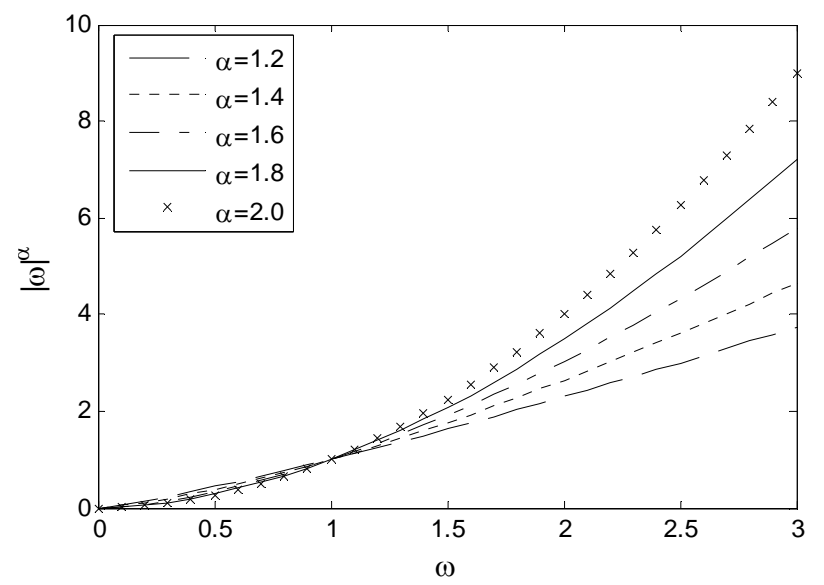

(b) $\alpha \in(1.0,2.0]$

Figure 3. Amplitude-frequency response

When $\alpha \in(0,1.0]$, in the extremely low frequency section, corresponding to images' smooth area, the attenuation degree of image contour resulted by fractional differential is better than the first order differential. In the medium frequency section, corresponding to images' texture area, the fractional order can protect more image details than the first order. In the high frequency section, corresponding to images' edge and noise area, the fractional order has obvious reduction function than the first order. When $\alpha \in(1.0,2.0]$, in the extremely low frequency section, the attenuation degree of image contour resulted by fractional differential is similar to the one obtained by the first order differential. In the medium frequency and the high frequency sections, the enhancement degree of image edge and noise with fractional differential is much better than the first order differential. Considering the details protection ability and the noise roustness, we select differential order $\alpha \in(0,1.0)$
B. The selection of fractional differential items number

In the following, we will analysis the influence of the fractional differential items number on image segmentation effect. Select a simulated brain MRI image as test image, the size is $258 * 258$, noise is $5 \%$, and intensity inhomogeneity is $20 \%$, shown in Fig.4 Let $\alpha=0.2, \tau=3$, Fig.5 shows the segmentation results of the fractional Sobel operator in $3 \times 3,4 \times 4,5 \times 5,6 \times 6$, and $7 \times 7$ template.

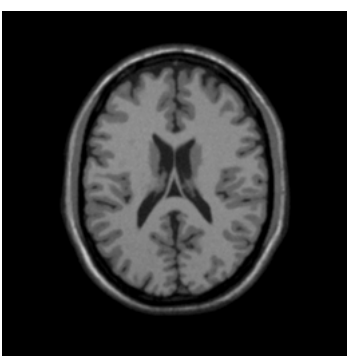

Figure 4. Test image

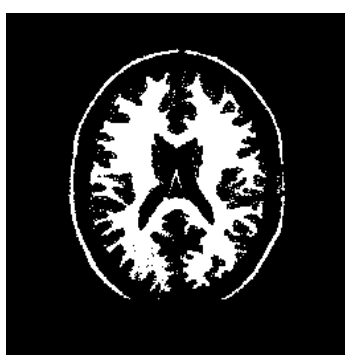

(a) $3 \times 3$
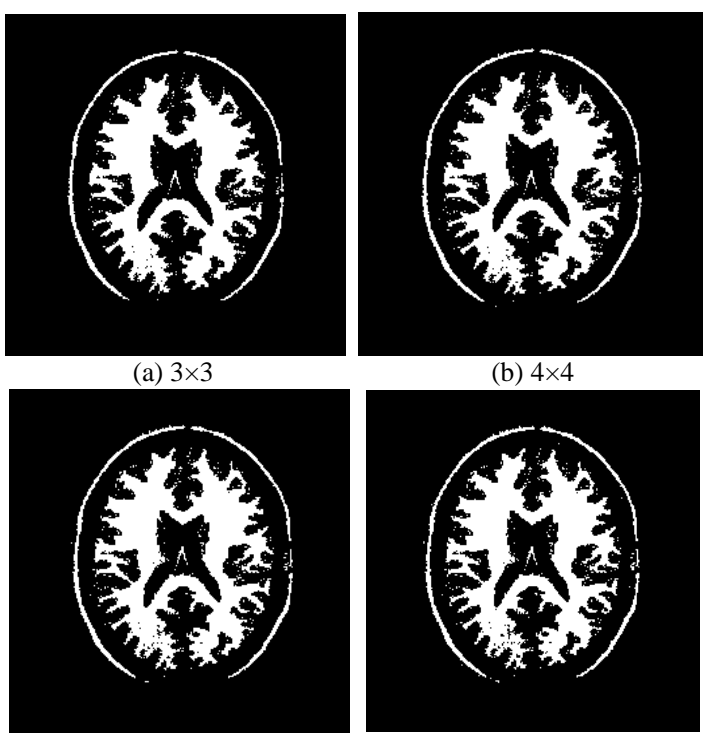

(c) $5 \times 5$

(d) $6 \times 6$

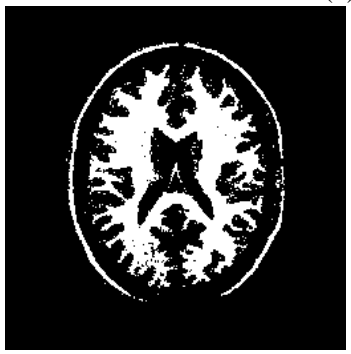

(e) $7 \times 7$

Figure 5. Segmentation results of fractional-order Sobel operator

Simulation results show that the fractional Sobel operator can segment image effectively, when it effect on the narrowband neighborhood of the image pixel. When the template is too large, the segmentation results can remain too much noise, and the segmentation processing need too long time.

\section{Performance analysis}

In the following, we provide numerical results to show the behavior of the proposed fractional- order Sobel 
operator. Let $k=3, \alpha$ be selected from 0 to 1 , take 0.1 as interval. We select Fig. 4 as test image. Let $\tau=3$. Fig. 6 lists the extraction results under different fractional order.

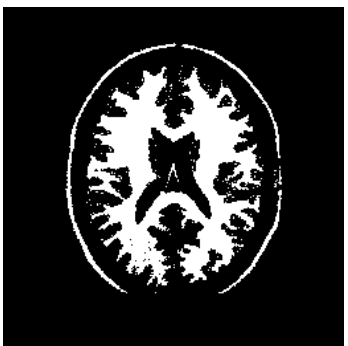

(a) $\alpha=0.2$

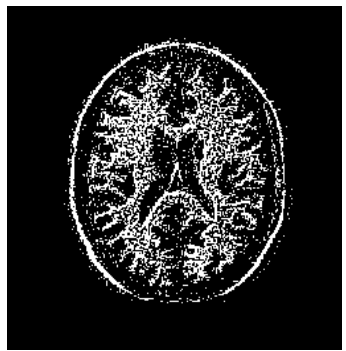

(c) $\alpha=0.6$

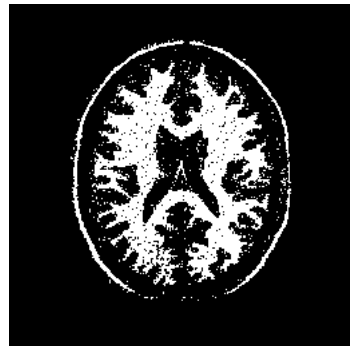

(b) $\alpha=0.4$

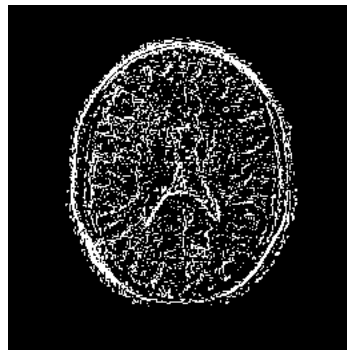

(d) $\alpha=0.8$

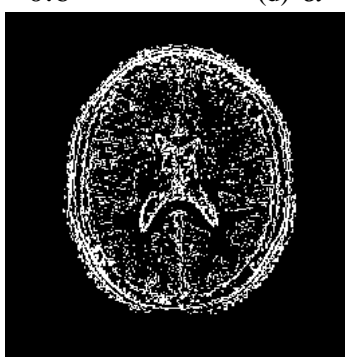

(e) $\alpha=1.0$

Figure 6. Comparison of segmentation results under different orders on a brain MRI image.

Experiment results show that the fractional-order Sobel operator is robust to noise and can restrain the influence of irrelevant detail compared with the Sobel operator. And the larger $\alpha$ is, the better image feature is preserved, but meanwhile the more noise is residual too.

\section{CONCLUSION}

From the definition, an important property can be observed that the integer derivative is a local operator while the fractional derivative is a global operator. The fractional operator can consider more neighboring information. Considering this characteristic, we generalize the traditional first-order Sobel edge detection operator to fractional-order for extracting image structure features. The novel operator has been validated on brain MR image with desirable performance.

\section{ACKNOWLEDGMENT}

The work is supported by the China National Nature Science Foundation (Grant No.61174145) and the China Liaoning Province Education Department Science and Technology Research Project (Grant No. L2013448).

\section{REFERENCES}

[1] M. E. Brummer, "Hough Transform Detection of the Longitudinal Fissure in Tomographic Head Images," IEEE Transactions on Medical Imaging, vol. 10, Jan. 1991, pp.74-81.

[2] X. Wang, "Laplacian Operator-Based Edge Detectors," IEEE Transactions on Pattern Analysis and Machine Intelligence, vol. 29, May. 2007, pp.886-890.

[3] S. A. Coleman, B. W. Scotney, and S. Suganthan, "Edge Detecting for Range Data Using Laplacian Operators,” IEEE Transactions on Image Processing, vol. 19, Nov. 2010, pp. 2814-2824.

[4] Y. F. Pu, J. L. Zhou, and X. Yuan, "Fractional Differential Mask: A Fractional Differential-Based Approach for Multiscale Texture Enhancement," IEEE Transactions on Image Processing, vol. 19, Feb. 2010, pp. 491-511.

[5] J. Bai and X. C. Feng, "Fractional-Order Anisotropic Diffusion for Image Denoising,” IEEE Transactions on Image Processing, vol. 16, Otc. 2007, pp. 2492-2502.

[6] N. Kanopoulos, N. Vasanthavada, R. Baker, "Design of an Image Edge Detection Filter Using the Sobel Operator,” IEEE J SolidState Circuits, vol. 23, 1988, pp.358-367.

[7] I. Zachevsky and Y. Y. Zeevi, "Single-Image Superresolution of Natural Stochastic Textures Based on Fractional Brownian Motion," IEEE Transactions on Image Processing, vol.23, Mar.2014, pp. 2096-2108.

[8] S. K. Rajput and N. K. Nishchal, "Image Encryption Based on Interference that Uses Fractional Fourier Domain Asymmetric Keys,” Applied Optics, vol. 51, Oct. 2012, pp. 1446-1452.

[9] V. Parot, C. Sing-Long, C. Lizama, C. Tejos, S. Uribe, and P. Irarrazaval, "Application of the Fractional Fourier Transform to Image Reconstruction in MRI,” Magnetic Resonance in Medicine, vol. 68, Jan. 2012, pp. 17-29.

[10] I. Odlubny, Fractional differential equations. New York: Academic Press, 1999. 